\title{
Plastic Soup: An Atlas of Ocean Pollution
}

By Michiel Roscam Abbing. 2019. Island Press. 136 pages, 27.00 CAD, Cloth or E-book.

Plastic is in almost every item we use and own. Its convenience as a relatively cheap and durable material has become rather inconvenient from an environmental standpoint. For a few years now I have personally tried to make my household less reliant on plastic and am continuously surprised at just how difficult it is. Whether it is pur-

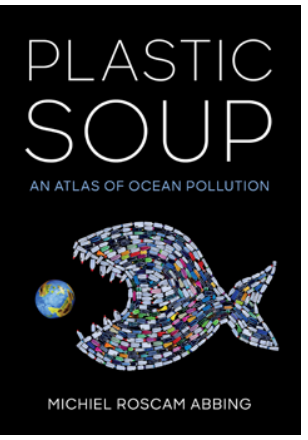
chasing food, soap, pet products, or craft supplies, it has been incredibly difficult to manoeuver in a world where things are not individually wrapped or contain plastic.

Plastic Soup: An Atlas of Ocean Pollution is a short book with a lot of impact, both by the written content and the visual content. Author Michiel Roscam Abbing is a political scientist actively working on the plastic soup problem since 2011 alongside the Plastic Soup Foundation. Trying to put an end to increasing amount of plastic pollution, the Plastic Soup Foundation works to tackle plastic issues at the source, something that is focussed on in this book.

Plastic Soup is separated into two distinct parts; the first, "On the Map", focusses on the plastic crisis: its creation, the effects plastic has on ecosystems and wildlife, and some of the major items contributing to the plastic problem. The second half of the book, "Off the Map", focusses on solving the plastic crisis, highlighting research and initiatives around the world, the introduction of laws, and even how art is bringing this issue into the forefront.

More than just an atlas of pollution, this book is also an atlas of hope. I found it so interesting to learn how many different stakeholders across the world are reducing the use of plastic. Being someone who loves food, the idea of lasered food to reduce packaging and stickers was especially of interest. This book also challenged the optics of plastic itself and some of the plastic solutions that are becoming popular. I found it interesting to read that, technically, plastic reduces food waste because it helps to extend the shelf life of many items. Similarly, it takes fewer emissions and less water to produce than paper does, another common packaging item. However, while this may seem like plastic is an obvious solution, the total lifecycle of the product says otherwise, emphasizing the need to think critically and in terms of lifecycles.

Critical thinking came up again in Chapter 7, "Between Belief and Hope", which tackles subjects such as recycling, bioplastics, and creating products out of ocean plastic. While these ideas may seem brilliant, they may be better than the actual results. Many books tend to focus on what you can do at an individual level and, while this book points out the roles our purchasing and lifestyle choices play, I was impressed by the author's emphasis on change at a level greater than a household. It helped me realize what more I can be doing at home and provided ideas I can push through to my local municipality and government.

I really enjoyed how in this first section the topics moved from obvious plastics, such as balloons, to plastics that we cannot see, such as microplastics. The author did a great job informing the reader of the problems and delivering the scientific evidence in a way that reaches all audiences. It was easy to understand, and the written content was enhanced by stunning photography and infographics. The format of the book makes it easy to read, providing a valuable tool for people seeking to learn more about the plastics issue. Throughout, heart-wrenching images bring home very effectively the message of the damage plastic has done to our planet and wildlife. Even if you don't read the book, the images alone will make you want to change your habits! I would recommend this book to anyone who is interested in learning more about plastics or for those, like me, who are trying to teach others and could use a resource jampacked with information.

TIANnA Burke

Conservation Biologist, Georgian Bay Biosphere Reserve, Parry Sound, ON 